\title{
Estimation of the Epidemiological Evolution Througha Modelling Analysis of the Covid-19 Outbreak
}

\section{Sanglier Contreras $\mathbf{G}^{\mathbf{1}}$, Robas Mora $\mathrm{M}^{\mathbf{2}}$ and Jimenez Gómez $\mathrm{P}^{\mathbf{2}}$}

${ }^{1}$ Department of Architecture and Design, Construction Engineering Area, Higher

Polytechnic School

${ }^{2}$ Microbiology Area, Pharmaceutical and Health Sciences Department, Faculty of

Pharmacy, Universidad San Pablo CEU, Boadilla Del Monte, Madrid, Spain

*Corresponding Author: Sanglier Contreras G, Department of Architecture and

Design, Construction Engineering Area, Higher Polytechnic School.
Received: February 26, 2020

Published: March 16, 2020

(C) All rights are reserved by Sanglier

Contreras G., et al.

\section{Abstract}

In December 2019, an outbreak of pneumonia of unknown origin began in Wuhan, China. The causative pathogen was identified as a new strain of coronavirus, COVID-19, similar to SARS-CoV. Since then and until today, epidemiological data confirm that It is spreading worldwide at a high rate.

Several vaccine strategies have been developed,but so far they have only been evaluated in animals. Currently, there is no specific antiviral therapy for $\mathrm{CoV}$ and the main treatments are supportive. In the same vein,easy transmissibility raises the scenario of massive spread.

From the branching processes (Galton-Watson process)which are discrete stochastic processes, a population is modeled that evolves in time and in each stage the process can take whole non-negative values, which will represent the total size of the population in that period. One of the tools to achieve these prediction and warning objectives consists of the mathematical modelling of the contagious processes, more specifically, the formulation of reliable indicators to evaluate their evolution overtime.

There are models to predict the evolution of each of the three populations by applying differential equations where certain boundary conditions are taken into account such as the variation of the model parameters according to some characteristics of the infection such as the infection rate,population size, duration of the infection period, etc. The present work deals with the development of models based on multi dimensional adjustment by means of polynomial equations assuming a linear dependence of the function with respect to each of the variables on which it depends in order to assess, in one way or another, the development of epidemics such as that of coronavirus (COVID- 19). Unless the mass distribution of an effective vaccine is achieved, the analysis and modeling of data yield results of great proportions.

Keywords: Estimation; Epidemiological; Througha Modelling

\section{Introduction}

Pneumonia is a type of acute respiratory infection. The alveoli of the lungs are inflated and often fill with pus and fluid, making breathing painful and limiting oxygen absorption. To date the most common viral infectious agents are Respiratory Syncytial Virus (RSV), Influenza, Parainfluenza, Adenovirus (less common) and
Measles. Unfortunately, this list may be altered by the recent appearance of COVID-19.

Viral pneumonia from COVID-19 is airborne. Symptoms of viral pneumonia may include coughing and/or shortness of breath, with or without fever, tachypnea or subcostal flushing, and often 
wheezing [1]. Very severely affected infants may be unable to eat or drink, and may experience loss of consciousness, hypothermia and seizures. Environmental factors also increase susceptibility to pneumonia [2]:

In December2019,an outbreak of pneumonia of unknown origin began in Wuhan, Hubei Province, China [1,3]. On December $8^{\text {th }}$ the first patient in Wuhan develops symptoms of corona virus $[4,5]$. OnJanuary $7_{\text {th }}$, Chinese authorities confirm that they have identified the virus as a new coronavirus. The first person dies of the virus on January 9, although his death was not announced until January $11_{\text {th }}$. On January 28 , the number of deaths exceeds 100 . The number of confirmed cases in mainland China exceeds the fatal outbreak of severe acute respiratory syndrome (SARS) in2003.

The causative pathogen was identified as a new strain of coronavirus similar to SARS- CoV. Although originally called Wuhan virus it has recently been renamed COVID-19 [5,6]. This virus is a "single-stranded RNA+" virus belonging to the family Corona viridae. Most coronaviruses cause only mild upper respiratory infections. However, cases of severe respiratory disease and outbreaks such as SARS- CoV or MERS-CoV are reported in the literature.

The WHO immediately declares a public health emergency of international concern. On February 6 the number of deaths exceeds 500. On February $11^{\text {th }}$, the number of deaths exceeds 1,000 worldwide. WHO names the coronavirus COVID-19.

At present, there is no specific antiviral therapy for $\mathrm{CoV}$ and the main treatments are supportive. Recombinant IFN with ribavirin has only limited effects against CoV infection [7]. After the SARS and MERS epidemics, great efforts have been devoted to the development of new antivirals targeting proteases, polymerases, MTases, and CoV entry proteins; however, none of them have been shown to be effective in clinical trials [8].

In addition, several vaccine strategies have been developed, such as the use of inactivated virus, live attenuated virus, viral vector-based vaccines, subunit vaccines, recombinant proteins and DNA vaccines, but so far, they have only been evaluated in animals [9].

More than $80 \%$ of patients have a mild illness with a high probability of recovery. In about $14 \%$ of cases, the virus causes serious illness, such as pneumonia and difficulty breathing. Approximately $5 \%$ of patients suffer from critical illness, such as respiratory failure, septic shock and multi-organ failure. In only $2 \%$ of reported cases is the virus fatal, and the risk of death increases as you age. Fortunately, so far, few cases are being reported among children. More research is needed to understand this uniqueness Since there is no effective therapy or vaccine, the best measures now are control of the source of infection, early diagnosis, notification, isolation, supportive treatments and timely publication of epidemic information to avoid unnecessary panic. For individuals, good personal hygiene, a tight mask, ventilation, and avoiding crowded places will help prevent $\mathrm{CoV}$ infection.

In some cases, epidemiology has used mathematical tools as an aid in prediction and effective health warning studies in short time periods in order to be able to estimate the evolution of future diseases.

The application of mathematical modeling of epidemics consists of the use of a particular language and mathematical tools to explain and predict the behavior of infectious and potentially harmful agents to human or animal populations.

There are mathematical models of deterministic, non-deterministic or branching processes that can help in the search for an adequate prediction in the face of a type of infection that determines

\section{Methodology}

For the present study of coronavirus propagation, updated data from the COVID- 19 Global Cases by John Hopkins CSSE have been used, the corresponding graphs have been represented and the curves have been adjusted, deriving the appropriate estimates in a mathematical way. Based on the equations obtained, predictions have been made in order to estimate the evolution of the epidemic.

The adjustment of the infected curves both in China and in the rest of the world, as well as of the cases of total deaths in the world, has been carried out using degree two and three polynomial equations, as will be seen below.

Curve fitting is a process by which, given a set of $\mathrm{N}$ pairs of points $\{x i, y i\}$ (where $\mathrm{x}$ is the independent variable and $\mathrm{y}$ the dependent one), a mathematical function $\mathrm{f}(\mathrm{x})$ is determined, so 
that the sum of the squares of the difference between the real image and the corresponding one obtained through the function fitted at each point is minimal:

$$
\varepsilon=\min \left(\sum_{i}^{N}\left(y_{i}-f\left(x_{i}\right)\right)^{2}\right)
$$

In most cases, a generic function $\mathrm{f}(\mathrm{x})$ is chosen as a function of one or more parameters and the value of these parameters is adjusted in such a way as to minimize the quadratic error, $\varepsilon$. The most typical form of this adjusted function is that of a polynomial of degree $\mathrm{G}$; obtaining for $\mathrm{G}=1$ a linear adjustment (or linearregression):

$f(x)=a_{0}+a_{1} x$

for $\mathrm{G}=2$ a parabolic fit:

$$
f(x)=a_{0}+a_{1} x+a_{2} x^{2}
$$

and so on.

On the other hand, it is possible to have a multi dimensional data set; that is, a set of $\mathrm{N}$ points in a $\mathrm{k}+1$-dimensional space of the type $\{x i(1), x i(2), \ldots, x i(k), \ldots y i$,$\} . Thefunction that will adjust to$ these points will be a function of variablek:

$$
y=f\left(x^{(I)}, x^{(2)}, \ldots, x^{(k)}\right)
$$

The simplest multi-dimensional adjustment is to assume a linear dependence of the function on each of the variables on which it depends; that is, by adjusting a function of the type:

$$
f\left(x^{(1)}, x^{(2)}, \ldots, x^{(k)}\right)=a_{0}+a_{1} x^{(1)}+a_{2} x^{(2)}+\ldots+a_{k} x^{(k)}
$$

in such a way that the quadratic error is minimized with respect to the set of parameters $\{a 0, a 1, \ldots, a k\}$. This is called multiline adjustment or regression used in forecasting the evolution of the coronavirus in this article.

It should be borne in mind that mathematical models are not sufficient to assess the origin and extent of an epidemic, and reliable data collection and appropriate treatment of the data are essential to extract the correctsolutions.

\section{Results}

For the evolution of confirmed cases of coronavirus infection in China between January the $20^{\text {th }}$ and February $12^{\text {th }}, 2020$ (24 days) a curve has been obtained, which is represented in graph1. Selected data has been collected in the Global Cases mentioned above, according to the following parameters: date, confirmed cases in China, other confirmed cases (rest of the world) and recovered individuals.

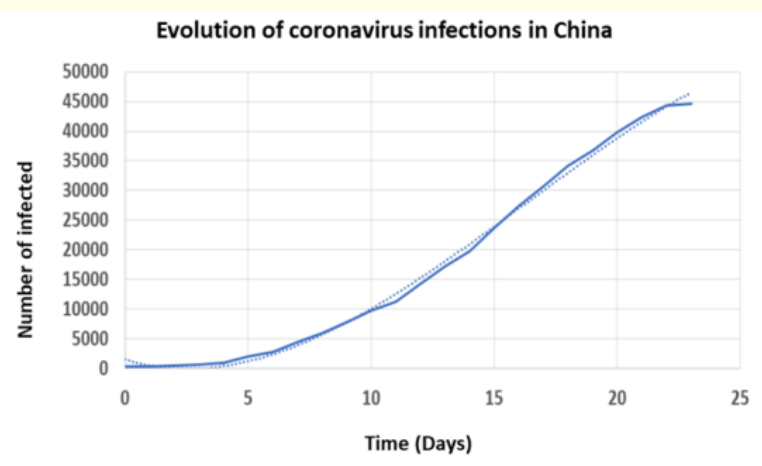

Graph 1: Evolution of the coronavirus in China.

This table shows the high evolution of the number of people infected by the coronavirus in China, especially from day five onwards.

The data curve has been adjusted to a third order or degree polynomial equation with a determination coefficient $\mathrm{R}^{2}$ very close to unity. This coefficient determines the quality of the model to reproduce the results, and the proportion of variation in the results that can be explained by the model.

$y=\left|-5,4589 x^{+}+265,17 x^{1}-1253,2 x+1518,61\right| 3^{1}=0,9973$

In the above equation, $\mathrm{Y}$ will be the number of infected and $\mathrm{X}$ the days elapsed.

The determination coefficient $\mathrm{R}^{2}$, defined between 0 and 1 ,gives an idea of the goodness of the fit, so that for values close to 1 the fit is perfect while for values close to zero it indicates the inexistence of a relationship between $\mathrm{x}$ and $\mathrm{y}$ with the proposed fit model. 
In the same way, the curve has been obtained for the case of those infected in other countries or the rest of the world. These results are represented in graph. 2.

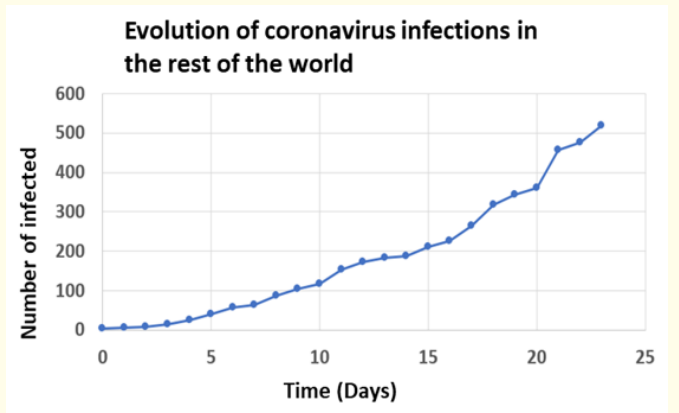

Graph 2: Evolution of coronavirus in the rest of the world except China.

The data curve has been adjusted to a second-degree polynomial with a coefficient of $\mathrm{R}^{2}$ determination very close to the unit.

$5=0,86966^{1}+1,64486+7,7865$

$3^{1}=0,9907$

As in the previous case, the $Y$ will correspond to the number of infected and the $\mathrm{X}$ to the days elapsed.

With respect to the number of deaths to date, graph 3 show the evolution of the data collected from the sources mentioned above.

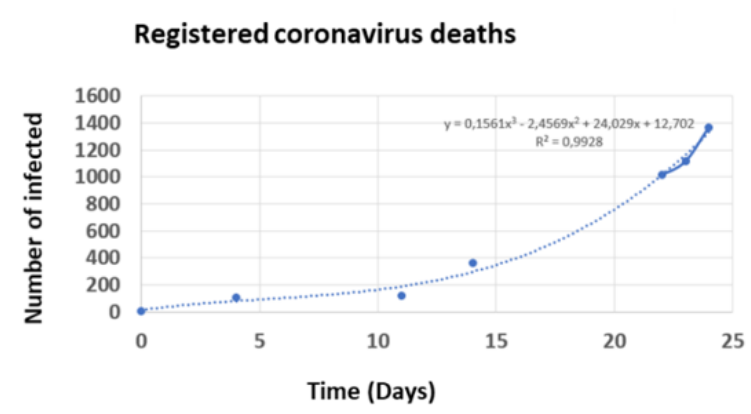

Graph 3: Evolution of deaths due to coronavirus.

The curve as in the previous cases has been modelled using a third-degree polynomial equation.

$5=0,15616^{+}-2,45696^{1}+24,0296+12,702$ with $3^{1}=0,9928$
The adjustment of the curve presents a determination coefficient, as in the previous cases, very close to unity.

The cumulative mortality rate data are shown in graph 4 below.

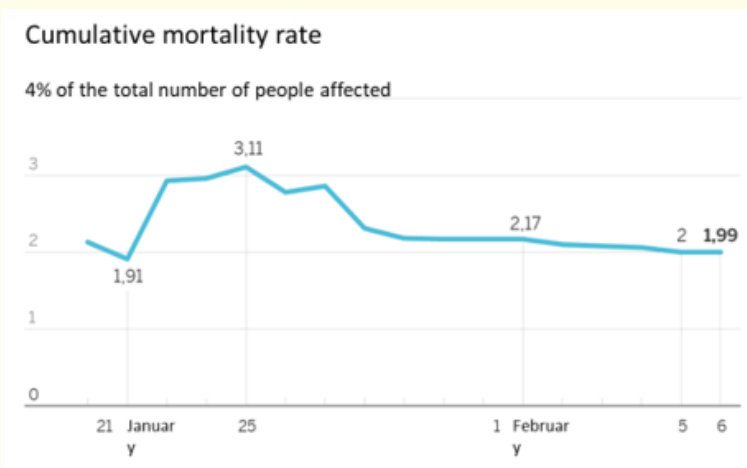

Graph 4: Mortality rate between the dates of January 20th, 2020 to February 12th, 2020 (24 days). Source: "El País" newspaper (February 2020).

Through mathematical modelling it has been predicted that the evolution of the coronavirus infection in the next 160 days (approximately three or four months) may present the following trend.

Graph 5 shows the evolution of an alarming growth in the number of infected people in China (the most populated country on Earth). The updated information of the United Nations $\left(\right.$ Statista $^{\odot}$ ) in 2019 on the number of people per continent (expressed in millions of individuals) was used as the basis for thecalculations.

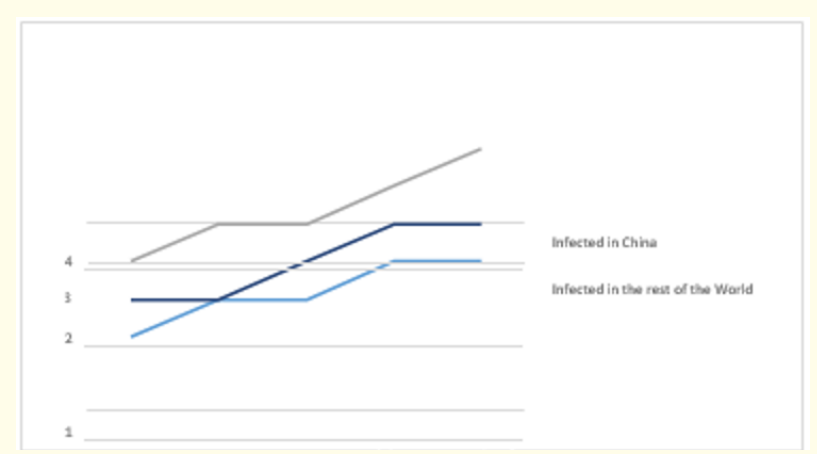

Graph 5: Prognosis of coronavirus evolution in the next 160 days (three to four months from February 2020), represented in logarithmic scale the number of affected individuals. 


\section{Discussion}

Viruses undergo, with certain periodicity, evolutionary changes. Viral genomes are subject to natural modifications by mutation or by recombination processes in the host, with the same frequency common to all nucleicacids [10]. Whenconditions are favorable to a particular new strain, it can be selected and become more epidemiologically relevant [11].

The emergence of a new strain may arise, inter alia, from the adaptation of an animal virus strain that acquires the ability to infect humans. In recent years, some studies have warned of the emergence of mutants of known viruses that could manifest new diseases and have the capacity to infect large groups of human populations $[12,13]$.

Many opinions have been expressed regarding the epidemiological evolution of the virus. For Jin-Hong; [14], based on SARS epidemiological information, the outbreak is expected to last at least three months. This outbreak is expected to have a greater amount of transmission than the MERS-CoV 2015. Regardless of its evolutionary origin, still to be clarified, COVID-19 is spreading rapidly around the world at highspeed.

In the same sense,the possibility of transmission by asymptomatic infected persons raises the scenario of a massive spread $[8,15]$.

COVID-19 is transmitted from person to person through respiratory droplets emitted When coughing or sneezing. There is also a possibility that the virus may exist and spread through contaminated fecal matter.

Concerns about how long the new coronavirus can survive on surfaces are growing, so much so that China's central bank has taken steps to thoroughly clean and destroy its cash, which changes hands several times a day, in an effort to contain thevirus.

It is not known exactly how long the new corona virus can remain on surfaces and objects contaminated with the potential to infect people.

Kampf., et al. [16] have found that these human coronaviruses, such as SARS and MERS, persist on inanimate surfaces, including those made of metal, glass, or plastic, for up to nine days if that surface has not been disinfected [16,17]. Cleaning with common household products can efficiently activate surface Disinfection with $62-71 \%$ ethanol, $0.5 \%$ hydrogen peroxide or $0.1 \%$ sodium hypochlorite Or bleach.

The drop in the cumulative mortality rate may be due mainly to two factors: the first is that at the beginning of a new epidemic, serious cases are the first to attract attention, while mild cases go unnoticed. This makes the mortality recorded in those first days higher; and the second, predictably with a lesser impact, is due to the fact that as more information is available about the virus and the disease, although there is no specific treatment, care for the sick and the life support measures applied can be improved, thus improving survival. Likewise, the change in the criteria of the Chinese authorities to consider the number of people infected may modify the forecasts of the evolution of the disease.

A starting point for the use of mathematical models to study the spread of epidemics was the SIR(Susceptible-Infected-Recovered)model developed in 1927 by Mc KendricK and Kermack [18]. The model studied a population in which an epidemic could develop and divided the population into threegroups:

- Individuals susceptible to contracting $\mathrm{S}(\mathrm{t})$ disease.

- Individuals infectedI $(\mathrm{t})$.

- Individuals recovered $\mathrm{R}(\mathrm{t})$, including those who overcome the disease as well as those who die fromit.

The flow of transitions from one group to another follows the scheme below:

$\mathrm{S} \longrightarrow \mathrm{I} \longrightarrow \mathrm{R}$

The population is considered closed for any time, that is to say, neither births nor migrations are taken into account, in such a way that

$N=S(t)+I(t)+R(t)$

The CRS model uses standard variables of the total population. The model is described by the following differential equations:

$89=-; 9<; \quad 8<=; 9<-\Upsilon<; \quad 83=\Upsilon<$

8: $\quad 8: 8:$ 
with the parameter $\beta$ being the infection rate (probability of a person becoming ill when in contact with an infected person) and $1 / \Upsilon$ being the average time of infection (for a single individual) [19,20-22]

The objective of the model was to predict the evolution of each of the three populations by applying three differential equations where certain boundary conditions are taken into account such as the variation of the model parameters according to some characteristics of the infection such as the infection rate, population size, duration of the infection period, etc.

The study of the system of equations made it possible to determine an $\mathrm{R}_{0}$ parameter to help in the study of being able to estimate the incidence of a certain epidemic. The model defines a threshold quantity called the basic reproduction number $R_{0}$ which is defined through the properties of the process and is the average number of secondary cases caused by the infection of a single person at the beginning of the disease, so that if it was below a critical value $\left(\mathrm{R}_{0}=1\right)$, the outbreak was controlled, while if $R_{0}>1$, the disease continued to spread. Ro values for this new corona virus outbreak have been estimated a taround 2.24 so that the disease continues tospread.

The main focus of corona virus detection has been identified in the country with the largest population (China) and in the most populated continent (Asia). This has resulted in the virus infection spreading faster to the other continents and the evolution of the infection has been triggered. Figure 1 clearly show this clearly.

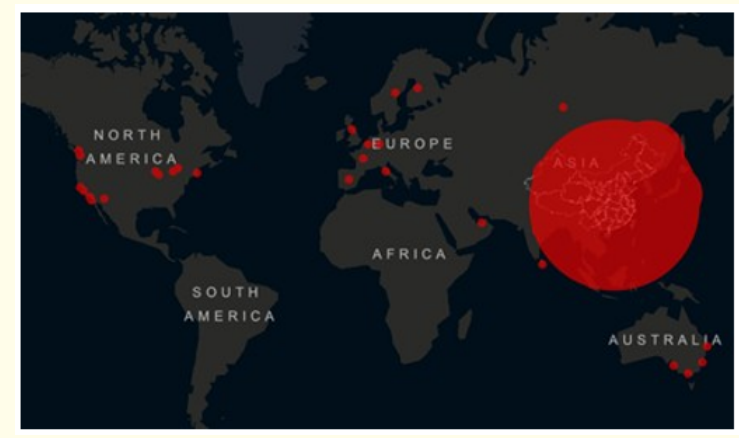

Figure 1 Evolution of the virus at a global level (COVID-19 Global Cases by John Hopkins CSSE).
At present, many versions appear with many different data on the evolution of this infection. This casts doubt on the veracity of the data provided by the Chinese government (Taiwan News). It is possible to think in the worst case scenario, if we observe all these exchanges of figures, that the number of infected and dead in China could be much higher (there is talk of 80 times more than officially give ndata),this would naturally trigger the forecasts made in this article and put humanity in danger. The news on this subject is very contradictory and could be a smear campaign against the Chinese government, according to the British Daily Mail. Due to all this, one should be cautious, but at the same time be prepared for more serious cases in the numbers of infected and dead.

Zao., et al. (2020) have confirmed that the initial growth phase followed a pattern of exponential growth. Under reporting is likely to have resulted in 469 (95\% CI 403-540) unreported cases from 1-15 January 2020. The reporting rate after 17 January 2020 is likely to have increased 21 times (95\% CI 18 -25) compared to the situation from 1-17 January 2020 on average. We estimate the R0 from COVID-19 at 2.56 (95\% CI 2.492.63).

\section{Conclusion}

Simple mathematical models such as those presented here take into account the evolution of the infection with all the conditions that the infection goes through, as mentioned in the methodology of this article. However, it would be desirable to develop more complex models that can predict, perhaps with greater accuracy, this type of disease.

The development or possession of more precise mathematical models does not remove the need for humanity to be on the alert for the threat of this type of virus, whether created by man or by nature itself. We must be prepared for this type of hreat.

According to the data on the prognosis of infection and death obtained in this article, efforts should be made to obtain a vaccine against this type of infection as soon as possible.

Significant monetary and tourism losses are beginning to be seen in the economic sector, mainly due to the questioning of the reliability of the epidemic data provided by the Chinese health authorities. This has created a climate of mistrust among society. 
The promotion of hygiene habits among the population, as well as the use of masks, as well as other types of infection control materials such as traditional products that allow disinfection, are questioned in terms of their effectiveness. This could lead to a mathematical modelling of the dynamics of the epidemic.

This article is intended to highlight the importance of investingational efforts with in our reach to control the outbreak, even before it extends to equally overpopulated countries such as India or the African continent.

The development of a safe and effective vaccine in time would be the most favorable solution to contain the outbreak and prevent the appearance of long chains of disease transmission.

\section{Bibliography}

1. Huang C., et al. "Clinical features of patients infected with 2019 novel coronavirus in Wuhan, China”. Lancet (2020).

2. World Health Organization WHO. (2019).

3. Zhu N., et al. "A novel coronavirus from patients with pneumonia in China, 2019". The New England Journal of Medicine (2020).

4. Wu P., et al. "Real-time tentative assessment of the epidemiological characteristics of novel coronavirus infections in $\mathrm{Wu}-$ han, China". Eurosurveillance (2020).

5. World Health Organization (a). WHO issues best practices for naming new human infectious (2020).

6. World Health Organization (b). Surveillance case definitions for human infection with novel coronavirus (nCoV) (2020).

7. CinatlJ., et al. "Treatment of SARS with human interferons". Lancet 362.9380 (2003): 293-294.

8. Chan JF., et al. "A familial cluster of pneumonia associated with the 2019 novel coronavirus indicating person-to-person transmission: a study of a family cluster". Lancet (2019).

9. Wit E., et al. "SARS and MERS: recent insights into emerging coronaviruses". Nature Reviews Microbiology 14.8 (2016): 523-534.

10. Hays JN. "Epidemics and Pandemics: Their Impacts on Human History (2005).

11. Cui J., et al. "Origin and evolution of pathogenic coronaviruses". Nature Reviews Microbiology 17.3 (2019):181-192.
12. Menachery VD., et al. "A SARS-like cluster of circulating bat coronaviruses shows potential for human emergence". Nature Medicine 21.12 (2015): 1508-1513.

13. ChanJF., et al. "Broad-spectrum antivirals for the emerging Middle East respiratory syndrome coronavirus". Journal of Infection 67.6 (2013): 606-616.

14. Jin-Hong Y. "The Fight against the 2019-nCoV Outbreak: An Arduous March Has Just Begun". Journal of Korean Medical Science 3 (2020): 354.

15. Munster VJ., et al. "A novel coronavirus emerging in China - key questions for impact assessment". The New England Journal of Medicine (2020).

16. Kampf G., et al. "Persistence of coronaviruses on inanimate surfaces and their inactivation with biocidal agents". Journal of Hospital Infection (2020).

17. Geller C., et al. "Human coronaviruses: insights into environmental resistance and its influence on the development of new antiseptic strategies". Viruses 4 (2012): 3044-3068.

18. Kermack WO And Mc Kendrick AG. "Contributions to the Mathematical Theory of Epidemics". Proceedings of the Royal Society $A 115$ (1927): 700-721.

19. Hethcote HW. "The mathematics of infectious diseases". Society for Industrial and Applied Mathematics 42 (2000): 599-653.

20. Daley DJ and Gani J. "Epidemic Modeling and Introduction”. NY: Cambridge University Press (2005).

21. Kermack WO and McKendrick AG. "Contributions to the Mathematical theory of Epidemics. III Further studies on the problem of endemicity". Proceedings of the Royal Society A (1933): 141-194.

22. Trottier H and Philippe P. "Deterministic modeling of infectious diseases: theory and methods". The Internet Journal of Infectious Diseases (2001).

\section{Assets from publication with us}

- Prompt Acknowledgement after receiving the article

- Thorough Double blinded peer review

- Rapid Publication

- Issue of Publication Certificate

- High visibility of your Published work

Website: https://www.actascientific.com/

Submit Article: https://www.actascientific.com/submission.php

Email us: editor@actascientific.com

Contact us: +919182824667 